\title{
CORRESPONDENCE
}

\section{Inhibitory effect of rose oil products on Helicobacter pylori growth in vitro: preliminary report}

Helicobacter pylori is now well established as the aetiological agent of chronic gastritis and peptic ulcer, as well as a risk factor for development of gastric malignancy $[1,2]$. Eradication of the organism leads to significant reduction in ulcer relapse, but is difficult to achieve because of non-compliance, side-effects and resistance to antimicrobial agents used in double or triple therapy [2]. There is a need for alternative or additional strategies for the treatment of $H$. pylori infection [3]. Rosanol contains rose oil as active substance in $1 \%$ aqueous solution; it is prepared with the solvent Cremophor EL (BASF, Germany). Geraniol is an aliphatic terpene alcohol and is the major compound $(75 \%)$ of rose oil. An earlier study showed a beneficial effect of Rosanol on gastric ulcers induced experimentally in rats [4]. The disappearance of both ulcer and inflammatory cell infiltrates has been observed. Rosanol has also demonstrated spasmolytic properties. Moreover this agent has shown antibacterial activity against several genera such as Pseudomonas, Staphylococcus and Escherichia [4]. The aim of this study was to evaluate for the first time the effect of Bulgarian rose oil preparations on $H$. pylori growth in vitro.

MICs of rose oil (Rosanol) 1\%, geraniol 1\% and Cremophor EL 5\% aqueous solutions were tested against $35 \mathrm{H}$. pylori isolates. Strains were adjusted to a turbidity equivalent to a McFarland 0.5 standard with sterile saline. Inocula of $10^{5}-10^{6} \mathrm{cfu}$ were transferred on to Brucella Agar (Merck) with sheep blood 5\% and Isovitalex (Becton Dickinson) 1\%, containing the following concentrations of active substances: rose oil and geraniol, $0.1-2 \mathrm{mg} / \mathrm{L}(10-200 \mathrm{ml} / \mathrm{L}$ of the solutions) and Cremophor EL, $0.5-10 \mathrm{mg} / \mathrm{L}(10-200 \mathrm{ml} /$ L). Control experiments were done in parallel on nonselective media. For disk susceptibility tests, the original bacterial suspensions were inoculated on to non-selective plates with swabs, and disks containing
$5 \mu \mathrm{l}$ of each solution (rose oil $1 \%$, geraniol $1 \%$ and Cremophor EL 5\%) were added. All plates were incubated micro-aerobically in a Campy Pak Plus (Becton Dickinson) at $37^{\circ} \mathrm{C}$ for 3 days. The MICs were defined as the lowest concentration of each agent needed to totally inhibit growth. Diameters of inhibitory zones were measured. To further evaluate the inhibitory effect of the rose oil preparations on five $H$. pylori strains, $10^{8} \mathrm{cfu}$ of each strain were pre-incubated in rose oil, geraniol or Cremophor EL solutions at concentrations of 3.3 and $5 \mathrm{mg} / \mathrm{L}$. Gram stains and subcultures on non-selective media were made after pre-incubation for $30 \mathrm{~min}$ and $18 \mathrm{~h}$ in micro-aerobic conditions at $37^{\circ} \mathrm{C}$.

Both rose oil and geraniol showed considerable activity against $H$. pylori. Median inhibitory zone diameters around the disks containing Rosanol and geraniol 1\% were similar (12.2 and $13.4 \mathrm{~mm}$ ) and larger than those induced by Cremophor EL (7.2 mm) (Table 1). H. pylori growth was inhibited by both rose oil and geraniol (MIC50 0.56 and $0.53 \mathrm{mg} / \mathrm{L}$ ) but was not affected by Cremophor EL (MIC90>10 mg/L). Rose oil and geraniol at concentrations of $2 \mathrm{mg} / \mathrm{L}$ inhibited the growth of $85.7 \%$ and $92 \%$ of the strains, respectively. Pre-incubation of $H$. pylori strains in both rose oil preparations for $30 \mathrm{~min}$ and $18 \mathrm{~h}$ induced coccoid forms in most $(>90 \%)$ of the bacterial cells in all strains tested. However, pre-incubation in Cremophor EL had no effect on spiral-shaped $H$. pylori and the viability of the organism was not affected. The inhibitory properties of Bulgarian rose oil preparations may be related to their major component of aliphatic alcohols. The MICs observed for rose oil and geraniol in this study were comparable to those of nitro-imidazoles [5].

In conclusion, Rosanol combined the following promising properties: inhibitory effect on $H$. pylori

Table 1. Inhibitory effects of rose oil, geraniol and Cremophor EL on $H$. pylori growth in vitro

\begin{tabular}{lccc}
\hline & & & \multicolumn{2}{c}{ Cremophor } \\
Preparation method & Rose oil & Geraniol & EL \\
\hline MIC (mg/L) & 35 & 25 & 13 \\
Number of strains & 0.56 & 0.53 & $>10$ \\
MIC50 & $>2$ & 1.75 & $>10$ \\
MIC90 & $0.1->2$ & $\leqslant 0.1->2$ & $2.5->10$ \\
Range & 35 & 19 & 19 \\
Disk diffusion* & 12.2 & 13.4 & 7.2 \\
Number of strains & $6-22$ & $7-20$ & $6-10$ \\
Median zone diameters (mm) & & & \\
Range of zone diameters (mm) & &
\end{tabular}

${ }^{*}$ Six-mm diameter disks were used. Disks contained $5 \mu \mathrm{l}$ of $1 \%$ rose oil (Rosanol), $1 \%$ geraniol or $5 \%$ Cremophor EL solutions. 
growth, anti-ulcer and spasmolytic activities, and lower cost in comparison with most antibacterial agents. However, it is not known whether the concentrations and the penetration of rose oil preparations in the gastric mucus could be sufficient to eradicate the bacteria. Further investigations are needed to evaluate whether Rosanol and geraniol may be useful, either alone or in combination with other agents, in the treatment of $H$. pylori infection.

Ludmila Boyanova and Gueorgui Neshev, Departments of Microbiology and Clinical Pharmacology, Medical University-Sofia, Sofia, Bulgaria.

\section{References}

1. Marshall BJ. Helicobacter pylori. Am J Gastroenterol 1994; 89: S116-S127.

2. Mégraud F. Resistance of Helicobacter pylori to antibiotics. Aliment Pharmacol Ther 1997; 11 Suppl 1: 43-53.

3. Petschow BW, Batema RP, Ford LL. Susceptibility of Helicobacter pylori to bactericidal properties of medium-chain monoglycerides and free fatty acids. Antimicrob Agents Chemother 1996; 40: 302-306.

4. Neshev G. Bulgarian rose oil - pharmacological and clinical studies. Thesis, Medical University-Sofia, Bulgaria, 1990.

5. Glupczynski Y. In vitro susceptibility testing of Helicobacter pylori to antimicrobial agents: basis for treatment or microbiologists' obsession. Int J Med Microbiol Virol Parasitol Infect Dis 1993; 280: 227-238. 